\title{
Rendimiento contable y EVA en la pyme de la industria del cuero, calzado y marroquinería en Colombia*
}

\author{
Jorge Alberto Rivera Godoy*
}

Fecha de recibido: 17 de octubre de 2018 Fecha de aprobado: 17 de mayo de 2019

Para citar: Rivera Godoy, J. A. (2020). Rendimiento contable y eva en la pyme de la industria del cuero, calzado y marroquinería en Colombia. Universidad \& Empresa, 22(38), 131-159. https://doi.org/10.12804/revistas.urosario.edu.co/empresa/a.7335

* $\quad$ Artículo resultado de un estudio realizado en el 2017 dentro de la línea de investigación de Evaluación del desempeño financiero de empresas del sector real en Colombia, que adelanta el Grupo de Investigación en Generación de Valor Económico de la Universidad del Valle (categoría C de Colciencias).

** Doctor Distinguido Cum Laude en Ciencias Económicas y Empresariales, Universidad Autónoma de Madrid, España. Especialista en Finanzas, Universidad del Valle, Cali, Colombia. Profesor titular, Universidad del Valle, Facultad de Ciencias de la Administración, Departamento de Contabilidad y Finanzas. Coordinador del grupo de investigación en Generación de Valor Económico (gigve) de la Universidad del Valle (categoría C de Colciencias). Correo electrónico: jorge.rivera@correounivalle.edu.co ORCID: http://orcid.org/0000-0003-2319-1674 


\section{Resumen}

Este artículo presenta los resultados de una investigación que tiene como propósito evaluar el desempeño financiero de la pyme del sector cuero, calzado y marroquinería en Colombia en el periodo 2010-2016, mediante un estudio que sigue como metodología el análisis de los indicadores contables y de valor económico agregado que miden su crecimiento, eficiencia, eficacia y efectividad.

Se encuentra que la pyme de este sector genera rendimientos contables en el periodo, siendo superior en la pequeña empresa por disponer de una mayor eficacia en la administración de costos de producción y ventas, una mayor eficiencia en la utilización de los activos y un uso de un mayor apalancamiento financiero positivo. Sin embargo, la pyme destruye valor económico agregado (EvA) en cada uno de los años; pero es la pequeña empresa la que menos destruye eva por cada unidad monetaria invertida. Esta destrucción de valor obedece a que el costo de recursos financieros es superior a la utilidad operacional después de impuestos. Estos hallazgos se fortalecerán con estudios similares para pymes más homogéneas en edad y ubicación geográfica.

Palabras clave: EVA, VMA, ROA, ROE, pymes del sector calzado, cuero y marroquinería.

\section{Accounting Performance and EVA in the SME of the Leather, Footwear and Leather Goods Industry in Colombia}

\section{Abstract}

This article presents the results of an investigation that aims to evaluate the financial performance of the SME of the leather, footwear, and leather goods sector in Colombia in the period 2010-2016, by means of a study that follows as a methodology the analysis of accounting indicators and economic value added that measure its growth, efficiency, and effectiveness. The research showed that this sector's SME generates accounting returns in the period, which are superior in small business because they have greater efficiency in the administration of production and sales costs, and greater efficiency in the use of the assets and positive financial leverage. smes destroy economic value added (EVA) in each of the years; however, small business is the one that least destroys EvA for each monetary unit invested. This destruction of value is due to the fact that the cost of financial resources is higher than the operating income after taxes. These findings will be strengthened with similar studies for more homogeneous smes in age and geographic location.

Keywords: EVA, VMA, ROA, ROE, SMEs footwear, leather, leather goods.

\section{Rendimento contável e EVA na PME da indústria do couro, calçado e marroquinaria na Colômbia}

\section{Resumo}

Este artigo apresenta os resultados de uma pesquisa que tem como propósito avaliar o desempenho financeiro da pME do setor couro, calçado e marroquinaria na Colômbia no período 2010-2016, mediante um estudo que segue como metodologia a análise dos indicadores contáveis e de valor econômico agregado que medem seu crescimento, eficiência, eficácia e efetividade.

Se encontra que a PME deste setor gera rendimentos contáveis no período, sendo superior na pequena empresa por dispor de uma maior eficácia na administração de custos de produção e vendas, uma maior eficiência na utilização dos ativos e um uso de maior alavancagem financeira positiva. No entanto, a PME destrói valor econômico agregado (EVA) em cada um dos anos; mas é a pequena empresa a que menos destrói EVA por cada unidade monetária invertida. Esta destruição de valor obedece a que o custo de recursos financeiros é superior à utilidade operacional depois de impostos.

Estes resultados se fortalecerão com estudos similares para PME mais homogêneas em idade e localização geográfica.

Palavras-chave: EVA, VMA, ROA, ROE, PME do setor calçado, couro e marroquinaria. 


\section{Introducción}

La industria de cuero, calzado y marroquinería ha sido seleccionada como uno de los 20 sectores estratégicos del Programa de Transformación Productiva del Ministerio de Comercio, Industria y Turismo, dado su crecimiento en la última década, su intensidad en mano de obra y a su alto potencial exportador, no solo por la firma de tratados de libre comercio, sino por la innovación y calidad de sus productos, lo que ha llevado a esta institución a crear un plan de negocios para este sector con visión 2028, con la colaboración de Fedecuero, Acicam y la Universidad del Rosario, entre otros (Programa de Trasformación Productiva, 2018). No obstante los avances en estrategias para ejercer un liderazgo regional, es poco lo que se sabe sobre su desempeño financiero y, en particular, si la pyme, que representa más del $67 \%$ de este sector, es rentable y creadora de valor económico, y por qué ocurre esto, siendo esta información de gran interés para la comunidad empresarial, estatal y académica. Como aporte a esta necesidad, se ha desarrollado una investigación que se sintetiza en el presente artículo, haciendo un análisis financiero para el periodo 2010-2016.

Para emprender este trabajo, se ha utilizado como metodología el análisis de indicadores contables tradicionales que miden el comportamiento financiero de empresas con ánimo de lucro, específicamente con los que se pueda evaluar el crecimiento, la eficiencia en el uso de recursos, la eficacia en control de costos y gastos para generar utilidades, que conceptualmente son tratados en textos de finanzas corporativas, como, por ejemplo, Ross, Westerfield y Jordan (2014) y Rivera (2004), como, también, la evaluación de indicadores de valor económico agregado y de valor de mercado agregado que hacen parte del nuevo modelo de gestión basada en valor, en el que la consultora internacional Stern Steward \& Co, creadora de la marca $\mathrm{EvA}^{\circledR}$, se destaca como uno de los impulsores más importantes (Martín \& Petty, 2001).

La evaluación de indicadores de gestión de valor se realiza para confirmar o rectificar los resultados de los indicadores contables, dado que estos últimos han sido cuestionados como medidores del desempeño financiero en las empresas (Milla, 2010). Las objeciones se fundamentan en que el paradigma de las finanzas modernas manifiesta que el objetivo fundamental de las organizaciones es crear valor, y que los indicadores contables no miden esta creación, sino su efectividad para generar utilidades contables, lo que podría 
dar lugar a que una empresa genere utilidades contables, pero destruya valor económico. Estos casos se han presentado en empresas y sectores industriales en países desarrollados y emergentes.

Como resultados de este trabajo de investigación, se encuentra que los activos y ventas de la pyme crecen; se obtienen rentabilidades contables, siendo superiores para la pequeña empresa que logra una mayor eficiencia en la utilización de los activos, hace un mayor uso de una palanca financiera positiva y es más eficaz en el control de sus costos de producción y venta.

Estos resultados favorables son contrarios a los encontrados mediante los indicadores de gestión: la pyme del sector cuero, calzado y marroquinería destruye eva en todos los años y, por consiguiente, el valor de mercado agregado en el periodo de análisis es negativo; además se encuentra que la mediana empresa destruye más valor económico agregado por unidad monetaria invertida. Estos hallazgos son contrastados con datos del desempeño financiero de toda la industria en Colombia.

El contenido del artículo se ha organizado de la siguiente forma: primero, se presenta la metodología, eligiendo y definiendo los indicadores financieros apropiados para este estudio. Luego, se muestran los principales resultados de otra investigación referente en el ámbito nacional. Después, se describe de forma breve la industria y la pyme del sector cuero, calzado y marroquinería en Colombia y se analizan los indicadores del desempeño financiero de la pyme de este sector en el septenio. Por último, se comparan y analizan estos resultados con los reportados en la fuente referente. Y se concluye sobre los principales descubrimientos de esta investigación.

\section{Metodología}

Para cumplir con el objetivo de esta investigación, se sigue como método el análisis estático y de tendencias de indicadores contables y de gestión del valor más apropiadas para la evaluación del desempeño financiero de pequeñas y medianas empresas de un sector económico. 
Los indicadores contables seleccionados son los de crecimiento, eficiencia, eficacia y efectividad, mientras que los indicadores de gestión del valor elegidos son el valor económico agregado, Eva y el valor de mercado agregado, vMA. Los indicadores de crecimiento miden el desarrollo del sector en relación con las ventas, activos y utilidades netas.

Los indicadores de eficiencia miden la intensidad con que las empresas utilizan sus activos para generar ventas (Wild, Subramanyam \& Hasley, 2007); entre los que sobresalen la rotación de la cartera, de los inventarios, de los activos fijos, de los activos operacionales y de los activos totales (Rivera, 2004).

Los indicadores de eficacia permiten medir el efecto que tienen los costos y gastos de las empresas sobre la utilidad, como sucede con los que influyen sobre los márgenes de utilidad bruta, operacional, operacional después de impuestos y neta (Rivera, 2004).

Los indicadores de efectividad miden los beneficios que recibe la empresa y sus propietarios por la inversión realizada; el primero, por medio del rendimiento del activo, ROA, y el segundo, mediante el rendimiento del patrimonio, roe. En el sistema Dupont se establece que el ROA resulta del producto entre la rotación de activos y el margen de utilidad operacional después de impuestos; mientras que con el sistema Dupont ampliado, el roE es el producto de la rotación de activos, el margen de utilidad neta y el apalancamiento financiero $^{1}$ (Ross, et al., 2014; Rivera, 2004).

El Eva es la utilidad residual resultante de restar de la utilidad operacional después de impuestos un cargo por la utilización del capital (Stewart, 2000), y puede expresarse con la siguiente ecuación:

$$
E V A_{t}=U O D I_{t}-\text { Cargo de capital }_{t^{\prime}}
$$

donde $A N O_{t}$ es la utilidad después de impuestos en el periodo $t$; y el cargo de capital en el periodo $t$ es igual a:

$$
\text { Cargo de capitalt }=\left(A N O_{t}\right)\left(K o_{t}\right) \text {, }
$$

1 Apalancamiento financiero = activo total bruto promedio / patrimonio promedio. 
siendo $A N O_{t}$ el activo neto operacional del periodo. La variable $A N O_{t}$ es la sumatoria del capital de trabajo neto operativo $K T N O_{t}$ y del activo fijo neto operacional $A F N O_{t}$ :

Cargo de capitalt $=A N O_{t}=K T N O_{t}+A F N O_{t}$,

El $K_{T N O}$ es la diferencia entre los activos corrientes y los pasivos corrientes sin costo explícito. El $A F N O_{t}$ resulta de restar a los activos fijos operacionales su depreciación. El es el costo de capital medio ponderado del periodo $t$; según Modigliani y Miller (1963), se obtiene así:

$$
K_{o t}=K e(1-L)+K i(1-t) L,
$$

Donde $K e$ es el costo del capital propio o costo de oportunidad. ${ }^{2} L$ es el nivel de endeudamiento que resulta de dividir la deuda con costo explícito entre el activo neto operacional, $A N O$. $K i$ es el costo de la deuda, pero dado que los intereses son deducibles de la base gravable de la empresa, el costo de la deuda después de impuestos queda expresado como $K i(1-t)$; la $t$ representa la tasa de impuestos de la empresa.

Stewart (2000) presenta esta segunda forma de calcular el EvA:

$$
E V A=\left(A N O_{t}\right)\left[\left(U A I I_{t}\right)(1-t) /\left(A N O_{t}\right)-\left(K O_{t}\right)\right]
$$

Donde $(U A I I)(1-t) /\left(A N O_{t}\right)$ es el rendimiento después de impuestos del activo neto operacional. A la diferencia entre el rendimiento después de impuestos del activo neto operacional $A N O$ y el costo de capital medio ponderado $\mathrm{Ko}_{t}$, se le conoce como el margen de utilidad o pérdida residual.

Al traer a valor presente el Eva de varios años, se obtiene el valor de mercado agregado del periodo evaluado; que se puede expresar de la siguiente forma:

$$
V M A=\sum_{j=1}^{j=\mathrm{n}} /(1+K o j)^{j}
$$

También se determina el $E V A$ que genera el capital propio, aplicando la siguiente ecuación:

EVA de capital propio $_{t}=\left(\right.$ Patrimonio $\left._{t-1}\right)(R O E-K e)$

2 Para determinar Ke se seguirá la metodología del CAPM de Pure Play explicada por Rivera \& Alarcón (2012), dado que las pymes que conforman este sector no cotizan en la bolsa de valores. 
Donde $(R O E-K e)$ es el margen de utilidad o pérdida residual del capital propio.

Los Eva del capital propio se pueden traer a valor presente, y se obtiene el valor de mercado agregado $E V A$ del capital propio del periodo evaluado; pudiéndose expresar así:

$V M A=\sum_{j=1}^{j=\mathrm{n}}$ EVA capital $_{\text {propio }} /(1+K e j)^{j}$

Se analiza la gestión del valor con el EVA y sus tres inductores: UODI, ANO, Ko, así como el Eva de capital propio y sus inductores Patrimonio, roE y Ke.

Adicionalmente, se examina el $E V A$ que genera cada unidad monetaria del $A N O$ mediante la relación $E V A / A N O$, como, también, la utilidad operativa después de impuestos que se requiere para crear una unidad monetaria de valor económico agregado, mediante el indicador $U O D I / E V A$.

\section{Revisión de literatura}

Al hacer una revisión de los trabajos afines a los objetivos de esta investigación, se halla un estudio de Rivera y Duque (s.d.) en el que encuentran que la industria de cuero, calzado y marroquinería (ICCM) presenta un crecimiento de los activos y activos entre el 2010-2016, interrumpido con una caída en el 2015, mientras la utilidad neta oscila en este periodo (tabla 1).

Tabla 1. Activos, ventas y utilidad neta promedio de la ICCM en Colombia

\begin{tabular}{lcccccccc}
\hline & \multicolumn{7}{c}{ Crecimiento (MM\$) } \\
\hline & $\mathbf{2 0 1 0}$ & $\mathbf{2 0 1 1}$ & $\mathbf{2 0 1 2}$ & $\mathbf{2 0 1 3}$ & $\mathbf{2 0 1 4}$ & $\mathbf{2 0 1 5}$ & $\mathbf{2 0 1 6}$ & Promedio \\
\hline Ventas & 7673 & 9978 & 10859 & 14780 & 18312 & 16429 & 29848 & 15411 \\
\hline Utilidad neta & 245 & 415 & 412 & 369 & 445 & 354 & 1064 & 472 \\
\hline Activos & 7641 & 9471 & 10074 & 13722 & 16325 & 14413 & 26210 & 13980 \\
\hline
\end{tabular}

Nota: MM\$ denota cifras en millones de pesos colombianos.

Fuente: Rivera y Duque (s.d., p. 11).

En este mismo septenio esta industria muestra un ROA promedio de $4.8 \%$ y un ROE promedio de $7.0 \%$, siendo ambos positivos en cada uno de los años. El comportamiento 
del ROA y el ROE durante este periodo lo determina el margen de la utilidad operacional después de impuestos. El roe fue superior al ROA durante todos los años, debido al efecto multiplicador del apalancamiento financiero sobre el margen utilidad neta que, siendo inferior al margen de utilidad operacional después de impuestos, logró incrementarse de manera positiva (tabla 2).

Tabla 2. Indicadores de efectividad de la IсcM en Colombia

\begin{tabular}{|c|c|c|c|c|c|c|c|c|}
\hline & 2010 & 2011 & 2012 & 2013 & 2014 & 2015 & 2016 & Promedio \\
\hline \multicolumn{9}{|c|}{ a. ROA: Sistema Du Pont } \\
\hline $\mathrm{ROA}(\%)$ & 3.3 & 5.4 & 5.0 & 3.6 & 4.8 & 4.0 & 7.7 & 4.8 \\
\hline $\begin{array}{l}\text { Margen de utilidad operacional des- } \\
\text { pués de impuestos (\%) }\end{array}$ & 3.3 & 5.1 & 4.7 & 3.3 & 4.3 & 3.5 & 6.8 & 4.4 \\
\hline Rotación de activos totales (veces) & 1.0 & 1.0 & 1.1 & 1.1 & 1.1 & 1.1 & 1.1 & 1.1 \\
\hline \multicolumn{9}{|c|}{ b. ROE: Sistema Du Pont Ampliado } \\
\hline ROE (\%) & 6.3 & 8.6 & 8.0 & 5.4 & 5.4 & 5.0 & 10.6 & 7.0 \\
\hline Margen de utilidad neta (\%) & 3.2 & 4.2 & 3.8 & 2.5 & 2.4 & 2.1 & 3.6 & 3.1 \\
\hline Rotación de activos totales (veces) & 1.0 & 1.0 & 1.1 & 1.1 & 1.1 & 1.1 & 1.1 & 1.1 \\
\hline Apalancamiento financiero (\%) & 197.0 & 195.6 & 196.4 & 199.7 & 196.8 & 203.1 & 262.3 & 207.3 \\
\hline
\end{tabular}

Fuente: Rivera y Duque (s.d., p. 12).

Aunque esta industria tuvo rendimientos positivos, destruyó valor económico en cada uno de los años, debido a que el costo de capital fue superior al rendimiento del activo neto operacional. El comportamiento del eva sigue una correlación negativa en relación con el costo de capital, excepto en 2013. En este periodo el vma a comienzos del año 2010 fue \$-2310 MM (tabla 3). El Eva del capital propio también es negativo en todos los años, esto se debe a que el roe es inferior al Ke (tabla 3).

Tabla 3. eva promedio por empresa de la ICcM en Colombia

\begin{tabular}{lcccccccc}
\hline & $\mathbf{2 0 1 0}$ & $\mathbf{2 0 1 1}$ & $\mathbf{2 0 1 2}$ & $\mathbf{2 0 1 3}$ & $\mathbf{2 0 1 4}$ & $\mathbf{2 0 1 5}$ & $\mathbf{2 0 1 6}$ & Promedio \\
\hline EVA (MM\$) & -292 & -100 & -217 & $-\mathbf{3 6 6}$ & -510 & $-\mathbf{7 3 3}$ & $-\mathbf{8 2 1}$ & $-\mathbf{4 3 4}$ \\
\hline ANO (MM\$) & 4290 & 5113 & 5659 & 8857 & 10716 & 9040 & 18965 & 8949 \\
\hline UODI/ ANO (\%) & 5.9 & 9.9 & 9.0 & 5.5 & 7.4 & 6.4 & 10.7 & 7.8 \\
\hline Ko (\%) & 12.7 & 11.9 & 12.8 & 9.7 & 12.1 & 14.5 & 15.0 & 12.7 \\
\hline VMA a 1-1-2010 (MM\$) & -1740 & & & & & & & \\
\hline EVA del capital propio (MM\$) & -372 & -319 & -441 & -503 & -880 & -1108 & -866 & -641 \\
\hline & & & & & & & & Continúa
\end{tabular}




\begin{tabular}{lcccccccc}
\hline & $\mathbf{2 0 1 0}$ & $\mathbf{2 0 1 1}$ & $\mathbf{2 0 1 2}$ & $\mathbf{2 0 1 3}$ & $\mathbf{2 0 1 4}$ & $\mathbf{2 0 1 5}$ & $\mathbf{2 0 1 6}$ & Promedio \\
\hline ROE $(\%)$ & 6.3 & 8.6 & 8.0 & 5.4 & 5.4 & 5.0 & 10.6 & 7.0 \\
\hline Ke $(\%)$ & 15.9 & 15.2 & 16.6 & 12.7 & 16.0 & 20.6 & 19.3 & 16.6 \\
\hline VMA capital propio 1-1-2010 (MM\$) & -2310 & & & & & & & \\
\hline
\end{tabular}

Nota: $\mathrm{MM} \$$ denota cifras en millones de pesos colombianos.

Fuente: elaboración propia con base en Rivera y Duque (s.d., pp.13-14) y SIREM (Superintendencia de Sociedades, 2017), Superintendencia Financiera (2017) y Damodaran (2017).

\section{Resultados}

En este apartado se muestra la caracterización de la industria de cuero, calzado y marroquinería en Colombia, como la evaluación del desempeño financiero de las pymes de este mismo sector.

\subsection{Caracterización de la industria de calzado, cuero y marroquinería en Colombia}

La industria de cuero, calzado y marroquinería incluye el curtido de cueros, recurtido y teñido de pieles; fabricación de calzado y la fabricación de artículos de viaje, bolsos de mano y artículos similares elaborados en cuero, y fabricación de artículos de talabartería y guarnicionería. De acuerdo con la clasificación ciru (Dane, 2012), se encuentra en la división 1511, 152 y 1512 dentro de la sección C de la industria manufacturera.

Esta industria ha mantenido un promedio de 374 establecimientos que representa el 4.0\% del parque industrial nacional, ha creado 21146 empleos que corresponde a $3.1 \%$ de la empleabilidad que ofrece el sector industrial y ha generado un promedio anual de $\$ 730971$ millones de valor agregado, es decir, el $0.9 \%$ del valor agregado promedio de toda la industria del país (tabla 4).

Tabla 4. Número de establecimientos y empleos, y valor agregado: promedio 2010-2016

\begin{tabular}{lcccccc}
\hline & $\begin{array}{c}\text { No. } \\
\text { Establecimientos }\end{array}$ & $\%$ & $\begin{array}{c}\text { No. } \\
\text { Empleos }\end{array}$ & $\%$ & $\begin{array}{c}\text { Valor agregado } \\
(\$)\end{array}$ & $\%$ \\
\hline Industria nacional & 9301 & 100 & 688749 & 100 & 79093248 & 100 \\
\hline Sector cuero, calzado y marroquinería & 374 & 4.0 & 21146 & 3.1 & 730971 & 0.9 \\
\hline
\end{tabular}

Nota: Cifras monetarias en millones de pesos (\$MM).

Fuente: elaboración propia con base en Dane (2017a y 2017b). 
Las pequeñas y medianas empresas dedicadas a la fabricación de cuero, calzado y marroquinería han representado en el periodo $2011-2016$ un promedio $67.4 \%$ de todo este sector, de las cuales el $54.7 \%$ son pequeñas y el $12.8 \%$ son medianas (tabla 5 ).

Tabla 5. Pyme promedio 2011-2016 del sector fabricación cuero, calzado y marroquinería

\begin{tabular}{lccccccc}
\hline & $\mathbf{2 0 1 1}$ & $\mathbf{2 0 1 2}$ & $\mathbf{2 0 1 3}$ & $\mathbf{2 0 1 4}$ & $\mathbf{2 0 1 5}$ & $\mathbf{2 0 1 6}$ & Promedio \\
\hline Pymes (\%) & 69.2 & 67.4 & 67.9 & 65.1 & 64.0 & 71.0 & 67.4 \\
\hline Pequeña (\%) & 58.0 & 54.4 & 56.2 & 52.9 & 51.1 & 55.5 & 54.7 \\
\hline Mediana (\%) & 11.2 & 12.9 & 11.6 & $12.3 \%$ & $13.0 \%$ & 15.6 & 12.8 \\
\hline
\end{tabular}

Fuente: elaboración propia con base en Dane (2017a y 2017b).

\subsection{Las pymes del sector cuero, calzado y marroquinería en Colombia}

Para el cálculo de los indicadores contables y los relacionados con la creación de valor, se tomaron los estados financieros de las pequeñas y medianas empresas del sector cuero, calzado y marroquinería ${ }^{3}$ de 2010 al 2016 de la base de datos SIREM (Superintendencia de Sociedades, 2017), de donde se obtuvo información de un promedio en el periodo de 47 empresas pequeñas (Рссм) у 43 medianas (мссм). ${ }^{4}$ En el Anexo se identifica cada una de ellas.

\subsubsection{Evaluación del crecimiento}

Se observa un aumento de los activos y ventas promedio de las pequeñas y medianas empresas en el periodo estudiado, solo se presentan caídas puntuales en las pequeñas empresas en los activos de 2011 y las ventas de 2013; entre tanto, las utilidades netas fluctúan. Como era de esperar, los valores absolutos de cada uno de los años y promedios del sexenio fueron superiores en las medianas empresas, donde los activos, ventas y utilidad neta promedio fueron de 7365 MM\$, 7993 MM\$ y 150 MM\$, respectivamente, mientras que en las pequeñas empresas estos indicadores fueron en su orden de 1530 MM\$, 2181 MM\$ y 46 MM\$ (tabla 6).

3 Con código industrial internacional uniforme (CIIU) C1511, C152 y C1512 (Dane, 2012).

4 El tamaño se determinó conforme al Artículo 2. ${ }^{\circ}$ de la Ley 905 de 2004 (Colombia, 2004). 
Tabla 6. Activos, ventas y utilidad neta promedio

\begin{tabular}{lcccccccc}
\hline & $\mathbf{2 0 1 0}$ & $\mathbf{2 0 1 1}$ & $\mathbf{2 0 1 2}$ & $\mathbf{2 0 1 3}$ & $\mathbf{2 0 1 4}$ & $\mathbf{2 0 1 5}$ & $\mathbf{2 0 1 6}$ & Promedio \\
\hline Pequeña & \multicolumn{7}{c}{ Crecimiento (MM\$) } \\
\hline Activos & 1235 & 1209 & 1246 & 1471 & 1683 & 1753 & 2114 & 1530 \\
\hline Ventas & 1488 & 1658 & 1701 & 1687 & 2048 & 2582 & 4106 & 2181 \\
\hline Utilidad neta & 28 & 16 & 45 & 43 & 0.7 & 61 & 128 & 46 \\
\hline Mediana & & & & & & & & \\
\hline Activos & 6577 & 6604 & 6642 & 6895 & 7844 & 8125 & 8869 & 7365 \\
\hline Ventas & 5599 & 6297 & 6893 & 8111 & 9282 & 9605 & 10162 & 7993 \\
\hline Utilidad neta & 92 & 165 & 146 & 174 & 217 & 181 & 74 & 150 \\
\hline
\end{tabular}

Nota: MM\$ denota cifras en millones de pesos colombianos.

Fuente: elaboración propia con base en SIREM (Superintendencia de Sociedades, 2017).

\subsubsection{Evaluación de la eficiencia}

La rotación de cada uno de los activos de la pequeña empresa se incrementa anualmente entre 2014 y 2016, después de haber caído en 2013, en los primeros años solo el inventario y el activo fijo habían presentado ascensos continuos. Mientras que en la mediana empresa se observa un aumento de la rotación de cartera en el transcurrir del septenio, un incremento no continuo de la rotación inventario, un aumento hasta 2013 de la rotación de activos fijos, para luego disminuir, dando como resultado un incremento de la rotación de activos totales hasta 2014 y luego un decrecimiento. Al comparar estos indicadores por tamaño de empresas, se encuentra que en todos los años la rotación de activos fijos es superior en la pequeña empresa, igual sucede con la rotación de activos, que solo entre 2012-2013 es igual al de la mediana empresa, como también, con la rotación de cartera donde solo es superada por la mediana empresa entre 20132014; mientras que la rotación inventarios fue más alta en la mediana empresa, excepto en 2016. Los promedios del periodo ratifican que la rotación de activos fijos (8.0), la rotación de los activos totales (1.4), la rotación de cartera (3.9) de la pequeña empresa supera a la mediana empresa que presentan rotaciones de estos activos de (5.4), (1.1) y (3.3), respectivamente, mientras que el promedio de la rotación inventarios de la medina empresa (3.9) es superior a la pequeña empresa (tabla 7). 
Tabla 7. Indicadores de eficiencia

\begin{tabular}{|c|c|c|c|c|c|c|c|c|}
\hline & 2010 & 2011 & 2012 & 2013 & 2014 & 2015 & 2016 & Promedio \\
\hline & \multicolumn{8}{|c|}{ Rotación (veces) } \\
\hline \multicolumn{9}{|l|}{ Pequeña } \\
\hline Rotación de cartera & 2.9 & 3.5 & 3.6 & 3.1 & 3.4 & 3.9 & 6.9 & 3.9 \\
\hline Rotación de inventario & 3.0 & 3.2 & 3.1 & 2.8 & 3.1 & 3.9 & 7.1 & 3.7 \\
\hline Rotación de activos fijos & 6.8 & 7.9 & 8.1 & 7.1 & 7.4 & 8.3 & 10.1 & 8.0 \\
\hline Rotación de activos totales & 1.2 & 1.4 & 1.4 & 1.2 & 1.3 & 1.5 & 2.1 & 1.4 \\
\hline \multicolumn{9}{|l|}{ Mediana } \\
\hline Rotación de cartera & 2.3 & 2.6 & 2.8 & 3.2 & 3.7 & 3.8 & 5.0 & 3.3 \\
\hline Rotación de inventario & 3.3 & 3.4 & 3.4 & 3.8 & 4.6 & 4.3 & 4.5 & 3.9 \\
\hline Rotación de activos fijos & 4.6 & 5.4 & 6.0 & 6.4 & 5.6 & 5.2 & 4.6 & 5.4 \\
\hline Rotación de activos totales & 0.9 & 1.0 & 1.0 & 1.2 & 1.3 & 1.2 & 1.2 & 1.1 \\
\hline
\end{tabular}

Fuente: elaboración propia con base en sIREM (Superintendencia de Sociedades, 2017).

Lo anterior deja como balance que la pequeña empresa es más eficiente en el uso de los recursos físicos, particularmente de los activos fijos, y seguido de la cartera, no obstante, la mediana empresa saca ventaja en la gestión de inventarios.

\subsubsection{Evaluación de la eficacia}

En la tabla 8 se puede observar que todos los índices de eficacia en el control de costos y gastos son positivos, lo que revela que los ingresos superan los diferentes costos y gastos. El margen bruto decae hasta el penúltimo año en la pequeña empresa, mientras que en la mediana empresa sube en la primera parte del septenio y luego decae hasta 2015. El margen operacional varía en la pequeña empresa, en tanto en la mediana empresa aumenta hasta 2013 y después se descuelga hasta 2016. El margen neto es variante, tanto en la pequeña como en la mediana empresa, pero con sentido contrario y menos profundo en las medianas empresas que oscila entre $2.6 \%$ y $1.0 \%$, mientras que en la pequeña empresa fluctúa entre $0.0 \%$ y $3.1 \%$.

El promedio del margen de utilidad bruta es superior en la pequeña empresa (26.4\%) en comparación con la mediana empresa (25.6\%), lo que demuestra que en el periodo de estudio la pequeña empresa es más eficaz en el control de los costos de producción y venta, pues saca una diferencia a la mediana empresa de $0.8 \%$; pero cuando se compara el promedio de la utilidad operacional de la pequeña (4.9\%) en relación con la mediana (5.3\%), la superioridad es de esta última que no solo reduce el $0.8 \%$ del margen bruto, 
sino que la aventaja con un $0.4 \%$ adicional, con lo que se puede inferir que la mediana empresa es más eficaz en el control de los gastos de administración y ventas.

Cuando se contrasta el promedio margen neto de la pequeña y mediana empresa se encuentra que es idéntica para ambas (1.9\%), lo que permite deducir que estas últimas son más eficaces en el control de otros gastos, incluyendo los financieros.

Estos resultados muestran que la empresa mediana es superada por la eficacia en el control de costos de producción y venta, y otros gastos, de la empresa pequeña, pero a su vez muestra una mayor eficacia en el control de los gastos de administración y venta.

Tabla 8. Indicadores de eficacia

\begin{tabular}{|c|c|c|c|c|c|c|c|c|}
\hline & 2010 & 2011 & 2012 & 2013 & 2014 & 2015 & 2016 & Promedio \\
\hline & \multicolumn{8}{|c|}{ Márgenes (\%) } \\
\hline \multicolumn{9}{|l|}{ Pequeña } \\
\hline Margen de utilidad bruta & 27.5 & 27.3 & 26.9 & 26.6 & 26.2 & 24.9 & 25.1 & 26.4 \\
\hline Margen de utilidad operacional & 5.5 & 4.5 & 5.3 & 3.2 & 3.3 & 5.7 & 6.5 & 4.9 \\
\hline Margen de utilidad neta & 1.8 & 1.0 & 2.7 & 2.5 & 0.0 & 2.4 & 3.1 & 1.9 \\
\hline \multicolumn{9}{|l|}{ Mediana } \\
\hline Margen de utilidad bruta & 24.1 & 25.9 & 27.3 & 27.2 & 25.7 & 23.7 & 25.1 & 25.6 \\
\hline Margen de utilidad operacional & 1.9 & 6.0 & 6.4 & 6.6 & 6.4 & 5.8 & 3.9 & 5.3 \\
\hline Margen de utilidad neta & 1.6 & 2.6 & 2.1 & 2.1 & 2.3 & 1.9 & 0.7 & 1.9 \\
\hline
\end{tabular}

Fuente: elaboración propia con base en SIREM (Superintendencia de Sociedades, 2017).

\subsubsection{Evaluación de la efectividad}

La empresa pequeña fue más efectiva en el logro de rendimientos sobre la inversión, esto se puede evidenciar al compararlo con la empresa mediana el ROA (4.8\% frente a $3.5 \%)$ y el roe (6.0\% frente a $3.7 \%)$ (tabla 9$)$.

El rendimiento del activo de la pequeña empresa fue superior a la mediana empresa en casi todos los años, excepto en 2013 y 2014, debido a su mayor eficacia en el manejo de los costos de producción y venta y de los impuestos, lo que le permitió mantener un mayor margen de utilidad operacional después de impuestos (3.3\% frente a 3.1\%), y a 
una mayor eficiencia en el uso de activos (rotación de 1.4 veces frente a 1.1 veces) (parte a y c de la tabla 9).

De otro lado, el rendimiento del capital propio de la pequeña empresa fue superior a las medianas empresas, salvo en 2011, 2013 y 2014. Los motivos de esta supremacía radican principalmente en que las pequeñas empresas fueron más eficientes en el uso de sus activos, y al uso de un mayor apalancamiento financiero, que para ambas categorías de empresas fue positivo (parte b y d de la tabla 9).

Tabla 9. Indicadores de efectividad

\begin{tabular}{|c|c|c|c|c|c|c|c|c|}
\hline & 2010 & 2011 & 2012 & 2013 & 2014 & 2015 & 2016 & Promedio \\
\hline \multicolumn{9}{|l|}{ Pequeña } \\
\hline & \multicolumn{8}{|c|}{ a. ROA: Sistema Du Pont } \\
\hline ROA (\%) & 5.3 & 4.1 & 4.7 & 1.8 & 2.1 & 6.1 & 9.3 & 4.8 \\
\hline $\begin{array}{l}\text { Margen de utilidad operacional } \\
\text { después de impuestos (\%) }\end{array}$ & 4.4 & 3.0 & 3.5 & 1.6 & 1.7 & 4.1 & 4.8 & 3.3 \\
\hline \multirow[t]{2}{*}{ Rotación de activos totales (veces) } & 1.2 & 1.4 & 1.4 & 1.2 & 1.3 & 1.5 & 2.1 & 1.4 \\
\hline & \multicolumn{8}{|c|}{ b. ROE: Sistema Du Pont Ampliado } \\
\hline ROE (\%) & 6.5 & 3.2 & 8.2 & 5.5 & 0.1 & 6.6 & 12.2 & 6.0 \\
\hline Margen de utilidad neta (\%) & 1.8 & 1.0 & 2.7 & 2.5 & 0.0 & 2.4 & 3.1 & 1.9 \\
\hline Rotación de activos totales (veces) & 1.2 & 1.4 & 1.4 & 1.2 & 1.3 & 1.5 & 2.1 & 1.4 \\
\hline Apalancamiento financiero (\%) & 289.4 & 238.6 & 225.2 & 191.0 & 203.1 & 189.8 & 201.4 & 219.8 \\
\hline \multicolumn{9}{|l|}{ Mediana } \\
\hline & \multicolumn{8}{|c|}{ c. ROA: Sistema Du Pont } \\
\hline $\mathrm{ROA}(\%)$ & -0.5 & 3.3 & 4.5 & 5.3 & 4.8 & 4.6 & 2.2 & 3.5 \\
\hline $\begin{array}{l}\text { Margen de utilidad operacional } \\
\text { después de impuestos (\%) }\end{array}$ & -0.5 & 3.4 & 4.3 & 4.5 & 4.1 & 3.9 & 1.9 & 3.1 \\
\hline \multirow[t]{2}{*}{ Rotación de activos totales (veces) } & 0.8 & 1.0 & 1.0 & 1.2 & 1.3 & 1.2 & 1.2 & 1.1 \\
\hline & \multicolumn{8}{|c|}{ d. ROE: Sistema Du Pont Ampliado } \\
\hline ROE $(\%)$ & 2.9 & 5.0 & 2.9 & 5.7 & 3.7 & 4.0 & 1.9 & 3.7 \\
\hline Margen de utilidad neta (\%) & 1.6 & 2.6 & 2.1 & 2.1 & 2.3 & 1.9 & 0.7 & 1.9 \\
\hline Rotación de activos totales (veces) & 0.8 & 1.0 & 1.0 & 1.2 & 1.3 & 1.2 & 1.2 & 1.1 \\
\hline Apalancamiento financiero (\%) & 207.2 & 201.4 & 198.7 & 197.4 & 183.6 & 192.8 & 229.2 & 201.5 \\
\hline
\end{tabular}

Fuente: elaboración propia con base en SIREM (Superintendencia de Sociedades, 2017). 


\subsubsection{EVA de las pequeñas y medianas empresas de la industria del cuero, calzado y marroquinería: periodo 2010-2016}

La pequeña empresa promedio de la industria colombiana de cuero, calzado y marroquinería destruyó valor económico agregado en todos los años de estudio. La destrucción vino en ascenso hasta 2014, a partir de 2015 cambió de tendencia y la destrucción comenzó a descender. El eva no mantiene una correlación positiva con la UODI, ni negativa con el cargo de capital. En promedio, se tuvo un eva anual de -78 MM\$ en el periodo (tabla 10).

El comportamiento de la uODI está direccionado por la tendencia de la utilidad operacional en todos los años, y a partir de 2011 con los impuestos (parte b de la tabla 10). La UAII presenta desde 2012 el mismo comportamiento de la utilidad bruta, y esta última sigue la misma tendencia de las ventas en todo el periodo, como también, del costo de venta y gastos de administración, excepto en 2013 (tabla 4 y parte b de la tabla 10).

El cargo de capital varía cada año, solo aumenta del 2014-2015. Se observa que su comportamiento está alineado con la fluctuación del costo de capital, ya que el ANO crece anualmente durante el septenio (parte a de la tabla 10).

El aumento del ANO es afín al comportamiento del KTNO, que sigue esta tendencia hasta el año 2015, y a la del AFNo, que solo se cae en 2012. La inversión en AFNo es inferior al KTNO (parte c de la tabla 10).

El comportamiento del Ko es similar a las fluctuaciones del Ke en todo el periodo. El promedio de Ko en el periodo fue de $15.0 \%$, que resulta de un Ke de 17.3\%, un Ki del 8.2\%, un endeudamiento del $29.0 \%$ y una tasa de impuestos del $29.0 \%$ (parte d de la tabla 10).

Al evaluar el margen de utilidad o pérdida residual, se observa que el uODI/ANo fue inferior al Ko en todos los años, aportando un motivo adicional por la cual la pequeña empresa de cuero, calzado y marroquinería destruye valor. La tendencia de UODI/ANO es similar al de la UODI/ventas, excepto en 2016, y al de ventas/ANo a partir de 2013 (tabla 10, parte e). 
Tabla 10. EVA promedio por pequeña empresa y sus inductores

\begin{tabular}{|c|c|c|c|c|c|c|c|c|}
\hline & 2010 & 2011 & 2012 & 2013 & 2014 & 2015 & 2016 & Promedio \\
\hline EVA (MM\$) & -52 & -53 & -59 & -88 & -126 & -100 & -69 & -78 \\
\hline UODI (MM\$) & 65 & 49 & 59 & 27 & 35 & 107 & 128 & 67 \\
\hline \multirow[t]{2}{*}{ Cargo de capital (MM\$) } & 117 & 103 & 118 & 115 & 161 & 208 & 198 & 146 \\
\hline & \multicolumn{8}{|c|}{ a. Inductores del cargo de capital } \\
\hline ANO (MM\$) & 700 & 727 & 750 & 1035 & 1165 & 1205 & 1239 & 974 \\
\hline \multirow[t]{2}{*}{ Ko (\%) } & 16.7 & 14.2 & 15.8 & 11.1 & 13.8 & 17.2 & 16.0 & 15.0 \\
\hline & \multicolumn{8}{|c|}{ b. Indicadores del inductor UODI } \\
\hline UAIl (MM\$) & 82 & 75 & 91 & 54 & 67 & 146 & 199 & 102 \\
\hline Impuestos (MM\$) & 17 & 25 & 32 & 27 & 32 & 39 & 70 & 35 \\
\hline Utilidad bruta (MM\$) & 409 & 453 & 458 & 449 & 537 & 643 & 1032 & 569 \\
\hline Costo de venta (MM\$) & 1079 & 1205 & 1243 & 1238 & 1511 & 1939 & 3074 & 1613 \\
\hline Gastos de Admón. (MM\$) & 183 & 216 & 223 & 224 & 301 & 330 & ND & 211 \\
\hline \multirow[t]{2}{*}{ Gastos de venta (MM\$) } & 144 & 162 & 143 & 172 & 168 & 166 & 264 & 174 \\
\hline & \multicolumn{8}{|c|}{ c. Indicadores del inductor ANO } \\
\hline KTNO (MM\$) & 417 & 446 & 477 & 709 & 715 & 742 & 715 & 603 \\
\hline \multirow[t]{2}{*}{ AFNO (MM\$) } & 284 & 280 & 274 & 326 & 450 & 463 & 524 & 372 \\
\hline & \multicolumn{8}{|c|}{ d. Indicadores del Inductor Ko } \\
\hline $\operatorname{Ke}(\%)$ & 20.4 & 17.1 & 18.5 & 12.4 & 16.2 & 20.0 & 16.8 & 17.3 \\
\hline Ki (\%) & 6.9 & 7.7 & 9.0 & 7.3 & 7.2 & 8.0 & 11.4 & 8.2 \\
\hline Deuda con costo explícito (MM\$) & 173 & 176 & 160 & 198 & 253 & 234 & 112 & 187 \\
\hline$L(\%)$ & 24.8 & 24.3 & 21.3 & 19.1 & 21.8 & 19.4 & 9.1 & 20.0 \\
\hline \multirow[t]{2}{*}{$\mathrm{t}(\%)$} & 33.00 & 33.00 & 33.00 & 25.00 & 25.00 & 25.00 & 25.00 & 29.00 \\
\hline & \multicolumn{8}{|c|}{ e. Sistema Dupont del índice UODI/ANO } \\
\hline UODI/ ANO (\%) & 9.3 & 6.8 & 7.8 & 2.6 & 3.0 & 8.9 & 10.4 & 7.0 \\
\hline UODI/Nentas (\%) & 4.4 & 3.0 & 3.5 & 1.6 & 1.7 & 4.1 & 3.1 & 3.1 \\
\hline \multirow[t]{2}{*}{ Ventas/Ano (veces) } & 2.1 & 2.3 & 2.3 & 1.6 & 1.8 & 2.1 & 3.3 & 2.2 \\
\hline & \multicolumn{8}{|c|}{ f. Valor del mercado agregado } \\
\hline VMA a 1-1-2010 (MM\$) & -309 & & & & & & & \\
\hline
\end{tabular}

Nota: MM\$ denota cifras en millones de pesos colombianos.

Fuente: elaboración propia con base en SIREM (Superintendencia de Sociedades, 2017), Superintendencia Financiera (2016) y Damodaran (2016).

El margen promedio de pérdida residual de la pequeña empresa fue de $-8.0 \%$, resultado de la diferencia entre la UODI/ANO promedio (7.0\%) y el Ko promedio $(15.0 \%)$.

El vma de la pequeña empresa de la industria de cuero, calzado y marroquinería al 1 de enero de 2010 fue de -309 MM\$, lo que significa que a pesar de que se generaron 
utilidades netas contables en cada uno de los años del septenio, destruyó valor (tabla 6 y tabla 10, parte f).

De otro lado, la mediana empresa promedio de la industria colombiana de cuero, calzado y marroquinería destruyó valor económico agregado en cada uno de los años del septenio. Hasta 2013 disminuyó gradualmente la destrucción de valor, pero a partir de ese año la tendencia fue aumentar la destrucción de valor. Se observa que el eva sigue una dirección semejante a la de la UODI, excepto para 2014. El promedio del Eva en el periodo fue de -513 MM\$ (tabla 11).

El comportamiento de la uODI está direccionado por las tendencias de la utilidad operacional y de los impuestos, excepto con este último en los años 2012 y 2016, donde sus variaciones son contrarias. La UAII presenta un comportamiento similar al de la utilidad bruta, como, también a los gastos de administración, excepto en 2011; mientras que los costos y gastos de ventas crecen continuamente, con una reducción de estos últimos en el año 2011 (parte b de la tabla 11).

El cargo de capital presenta altibajos, su comportamiento mantiene una correlación positiva con Ko, y excepto por el año 2014 su correlación es negativa con relación al ANO (parte a de la tabla 11).

La dirección del ANO se encuentra explicado por el comportamiento del KTNO y del AFNO que oscilan en sentido contrario durante todo el septenio. Se observa que el KTNO es superior al AFNo (parte c de la tabla 11).

Los inductores del Ko muestran que su comportamiento se alinea con la fluctuación del Ke, la Ki también oscila, pero salvo los primeros años, en sentido contrario. El promedio de Ko en el periodo fue de 14.2\%, que resulta de un Ke de 16.5\%, un Ki del 8.2\%, un endeudamiento del $20.0 \%$ y una tasa de impuestos del $35.8 \%$ (parte d de la tabla 11).

Al evaluar el porcentaje de utilidad residual, se encuentra que UODI/ANO fue menor a Ko en cada uno de los años, esto confirma el motivo por el cual la mediana empresa de la industria de cuero, calzado y marroquinería destruye valor en todos los años evaluados. La tendencia de UODI/ANO es similar a la relación UODI/ventas: con un aumento hasta el año 
2013, y reducción en los siguientes años, y al indicador ventas/ANo que aumenta hasta el año 2013 (tabla 11, parte e).

Tabla 11. EVA promedio por mediana empresa y sus inductores

\begin{tabular}{|c|c|c|c|c|c|c|c|c|}
\hline & 2010 & 2011 & 2012 & 2013 & 2014 & 2015 & 2016 & Promedio \\
\hline EVA $(\mathrm{MM} \$)$ & -746 & -473 & -438 & -190 & -409 & -623 & -710 & -513 \\
\hline UODI (MM\$) & -29 & 216 & 297 & 363 & 377 & 374 & 197 & 257 \\
\hline \multirow[t]{2}{*}{ Cargo de capital (MM\$) } & 716 & 689 & 736 & 553 & 785 & 998 & 907 & 769 \\
\hline & \multicolumn{8}{|c|}{ a. Inductores del cargo de capital } \\
\hline ANO (MM\$) & 4828 & 4961 & 4986 & 5055 & 5984 & 5962 & 6027 & 5401 \\
\hline \multirow[t]{2}{*}{ Ko (\%) } & 14.8 & 13.9 & 14.7 & 11.0 & 13.1 & 16.7 & 15.0 & 14.2 \\
\hline & \multicolumn{8}{|c|}{ b. Indicadores del inductor UODI } \\
\hline UAll (MM\$) & 104 & 381 & 443 & 537 & 593 & 556 & 395 & 430 \\
\hline Impuestos (MM\$) & 133 & 165 & 146 & 174 & 217 & 181 & 198 & 173 \\
\hline Utilidad bruta (MM\$) & 1350 & 1632 & 1884 & 2206 & 2387 & 2279 & 2550 & 2041 \\
\hline Costo de venta (MM\$) & 4249 & 4665 & 5009 & 5905 & 6895 & 7326 & 7613 & 5952 \\
\hline Gastos de Admón. (MM\$) & 621 & 685 & 749 & 824 & 806 & 778 & 313 & 682 \\
\hline \multirow[t]{2}{*}{ Gastos de venta (MM\$) } & 625 & 566 & 692 & 845 & 988 & 945 & 1370 & 861 \\
\hline & \multicolumn{8}{|c|}{ c. Indicadores del inductor ANO } \\
\hline KTNO (MM\$) & 2587 & 2831 & 3024 & 3070 & 2729 & 3131 & 2881 & 2893 \\
\hline \multirow[t]{2}{*}{ AFNO (MM\$) } & 2241 & 2131 & 1963 & 1985 & 3255 & 2831 & 3146 & 2507 \\
\hline & \multicolumn{8}{|c|}{ d. Indicadores del Inductor Ko } \\
\hline $\operatorname{Ke}(\%)$ & 16.8 & 15.5 & 16.9 & 12.6 & 15.4 & 20.1 & 17.9 & 16.5 \\
\hline Ki (\%) & 6.9 & 7.7 & 9.0 & 7.3 & 7.2 & 8.0 & 11.4 & 8.2 \\
\hline Deuda con costo explícito (MM\$) & 762 & 786 & 992 & 1100 & 1251 & 1355 & 1.405 & 1093 \\
\hline$L(\%)$ & 15.8 & 15.8 & 19.9 & 21.7 & 20.9 & 22.7 & 23.3 & 20.0 \\
\hline \multirow[t]{2}{*}{$t(\%)$} & 33.0 & 33.0 & 33.0 & 32.0 & 37.0 & 33.0 & 50.0 & 35.8 \\
\hline & \multicolumn{8}{|c|}{ e. Sistema Dupont del índice UODI/ANO } \\
\hline UODI/ ANO (\%) & -0.6 & 4.4 & 6.0 & 7.2 & 6.3 & 6.3 & 3.3 & 4.7 \\
\hline UODI/Nentas (\%) & -0.5 & 3.4 & 4.3 & 4.5 & 4.1 & 3.9 & 1.9 & 3.1 \\
\hline \multirow[t]{2}{*}{ Ventas/Ano (veces) } & 1.2 & 1.3 & 1.4 & 1.6 & 1.6 & 1.6 & 1.7 & 1.5 \\
\hline & \multicolumn{8}{|c|}{ f. Valor del mercado agregado } \\
\hline VMA a 1-1-2010 (MM\$) & -2198 & & & & & & & \\
\hline
\end{tabular}

Nota: $\mathrm{MM} \$$ denota cifras en millones de pesos colombianos.

Fuente: elaboración propia con base en SIREM (Superintendencia de Sociedades, 2017), Superintendencia Financiera (2016) y Damodaran (2016).

El porcentaje promedio de pérdida residual de la mediana empresa fue de $-9.5 \%$, resultado de la diferencia entre la UODI/ANO promedio (4.7\%) y el Ko promedio (14.2\%). 
El vma de la mediana empresa de cuero, calzado y marroquinería al 1 de enero de 2010 fue de -2.198 MM\$, lo que demuestra que, a pesar de que generó utilidades netas contables en cada uno de los años, destruyó valor en todos ellos, y por consiguiente en todo el septenio (tabla 6 y tabla 11, parte f).

Tanto la РСCM como la MCCM en Colombia muestran EvAs negativos en cada uno de los años y, por consiguiente, un vma negativo en todo el septenio. En términos absolutos, estos indicadores son mayores en мссм, dado su mayor inversión en ANo (en promedio el ANo en PсCм fue de 974 MM\$, mientras que en la MCCD fue de 5401 MM\$), y se corrobora cuando se compara en términos relativos: el promedio del Eva/Ano de la РсCм fue de -0.08 y la de la мсCм fue de -0.10 (tabla 12). La pequeña empresa destruye menor eva por cada peso de ANo invertido porque su margen de pérdida residual fue de $-8.0 \%$, menor al de la MccD que fue de $-9.5 \%$.

Además, la Рссм debe generar más uODI que la мссм para destruir un peso de EvA, esto se puede ver si se compara el índice UODI/Eva promedio de todos los años de la PCCM (-1.0) con el de la мссм que fue de -0.7 (tabla 12).

Tabla 12. Otros indicadores de control del EVA

\begin{tabular}{lcccccccc}
\hline & $\mathbf{2 0 1 0}$ & $\mathbf{2 0 1 1}$ & $\mathbf{2 0 1 2}$ & $\mathbf{2 0 1 3}$ & $\mathbf{2 0 1 4}$ & $\mathbf{2 0 1 5}$ & $\mathbf{2 0 1 6}$ & Promedio \\
\hline Pequeña & & & & & & & & \\
\hline EVA/ANO & -0.07 & 0.07 & -0.08 & -0.09 & -0.11 & -0.08 & -0.06 & -0.08 \\
\hline UODI/EVA & -1.3 & -0.9 & -1.0 & -0.3 & -0.3 & -1.1 & -1.8 & -1.0 \\
\hline Mediana & & & & & & & & \\
\hline EVA/ANO & -0.15 & -0.10 & -0.09 & -0.04 & -0.07 & -0.10 & -0.12 & -0.10 \\
\hline UODI/EVA & 0.0 & -0.5 & -0.7 & -1.9 & -0.9 & -0.6 & -0.3 & -0.7 \\
\hline
\end{tabular}

Superintendencia Financiera (2016) y Damodaran (2016).

Fuente: elaboración propia con base en SIREM (Superintendencia de Sociedades, 2017.

Cuando se evalúa el Eva de capital propio de la РССм, se observa que es negativo en cada año con tendencia a mejorar desde 2011, aunque con una recaída en el 2014. Este comportamiento es muy afín al porcentaje de pérdida residual de capital propio, que en promedio fue de $-\mathbf{1 1 . 3} \%$ (resultado de la diferencia de un roe de $6.0 \%$ y un Ke del $17.3 \%$ ). Mientras que en la мссм el еva del capital propio también es negativo, pero fluctuante; y en varios años con una correlación positiva con el porcentaje de pérdida residual del capital propio, que en promedio fue de $-12.8 \%$, con un RoE del 3.7\% y un Ke del 16.5\% (tabla 13). 
Tabla 13. EVA promedio por empresa, generado por el capital propio

\begin{tabular}{lcccccccc}
\hline & $\mathbf{2 0 1 0}$ & $\mathbf{2 0 1 1}$ & $\mathbf{2 0 1 2}$ & $\mathbf{2 0 1 3}$ & $\mathbf{2 0 1 4}$ & $\mathbf{2 0 1 5}$ & $\mathbf{2 0 1 6}$ & Promedio \\
\hline Pequeña & & & & & & & & -18 \\
\hline EVA del capital propio (MM\$) & -59 & -70 & -57 & -53 & -133 & -123 & -48 & 12,2 \\
\hline ROE (\%) & 6,5 & 3.2 & 8.2 & 5.5 & 0.1 & 6.6 & 6.0 \\
\hline Ke (\%) & 20.4 & 17.1 & 18.5 & 12.4 & 16.2 & 20.0 & 16.8 & 17.3 \\
\hline VMA capital propio 1-1-2011(MM\$) & -309 & & & & & & & \\
\hline Mediana & & & & & & & \\
\hline EVA del capital propio (MM\$) & -439 & -345 & -469 & -241 & -499 & -676 & -168 & -405 \\
\hline ROE (\%) & 2.9 & 5.0 & 2.9 & 5.7 & 3.7 & 4.0 & 1.9 & 3.7 \\
\hline Ke (\%) & 16.8 & 15.5 & 16.9 & 12.6 & 15.4 & 20.1 & 17.9 & 16.5 \\
\hline VMA capital propio1-1-2011(MM\$) & -1642 & & & & & &
\end{tabular}

Nota: $\mathrm{MM} \$=$ cifras en millones de pesos colombianos.

Fuente: elaboración propia con base en SIREM (Superintendencia de Sociedades, 2017), Superintendencia Financiera (2016) y Damodaran (2016).

El vмA del capital propio fue negativo tanto para РРСм (-309 MM\$), como para МРСм (- 1642 MM).

\section{Discusión}

En este aparte se analizan y contrastan los principales resultados de esta investigación sobre las pequeñas y medianas empresas que fabrican cuero, calzado y marroquinería en Colombia con toda la industria de este sector, haciendo uso de los indicadores financieros relacionados con el crecimiento, eficiencia, eficacia, efectividad y el valor económico agregado.

\subsection{Crecimiento}

Los valores promedio de ventas, activos y utilidad de la РСсм y la мссм encontrados en este estudio son inferiores a los que se muestran en la ICCM, esto debido a que este último no solo considera las pymes, sino también las grandes empresas de este sector en Colombia. La tendencia creciente de las ventas y activos se ha presentado tanto para la ICCM, como para las РССм у мССм, donde solo las caídas de estos indicadores en el año 2015 en la IсСм, los activos en el 2011 y las ventas en el 2013 en la РССм se separaron de esta dirección; mientras que la utilidades netas en los tres grupos es cambiante (tabla 14). 
Tabla 14. Comparación de los indicadores de crecimiento promedio del sector

\begin{tabular}{|c|c|c|c|c|c|c|c|c|}
\hline Indicador & 2010 & 2011 & 2012 & 2013 & 2014 & 2015 & 2016 & Promedio \\
\hline & \multicolumn{8}{|c|}{ PCCM } \\
\hline Ventas (MM\$) & $1 . .488$ & 1658 & 1701 & 1687 & 2048 & 2582 & 4106 & 2181 \\
\hline Activos (MM\$) & $1 . .235$ & 1209 & 1246 & 1471 & 1683 & 1753 & 2114 & 1530 \\
\hline \multirow[t]{2}{*}{ Utilidad neta (MM\$) } & 28 & 16 & 45 & 43 & 0,7 & 61 & 128 & 46 \\
\hline & \multicolumn{8}{|c|}{ MCCM } \\
\hline Ventas (MM\$) & 5599 & 6297 & 6893 & 8111 & 9282 & 9605 & 4106 & 7128 \\
\hline Activos (MM\$) & 6577 & 6604 & 6642 & 6895 & 7844 & 8125 & 8869 & 7365 \\
\hline \multirow[t]{2}{*}{ Utilidad neta (MM\$) } & 92 & 165 & 146 & 174 & 217 & 181 & 74 & 150 \\
\hline & \multicolumn{8}{|c|}{ ICCM } \\
\hline Ventas (MM\$) & 7673 & 9978 & 10859 & 14780 & 18312 & 16429 & 29848 & 15411 \\
\hline Activos (MM\$) & 7641 & 9471 & 10074 & 13722 & 16325 & 14413 & 26210 & 13980 \\
\hline Utilidad neta (MM\$) & 245 & 415 & 412 & 369 & 445 & 354 & 1064 & 472 \\
\hline
\end{tabular}

Nota: $\mathrm{MM} \$$ denota cifras en millones de pesos colombianos.

Fuente: elaboración propia con base en las tablas 1 y 6.

\subsection{Efectividad}

Al cotejar el roA promedio de la Іссм (4.8\%), la Рссм (4.8\%) y la мссм (3.5\%) se aprecia que toda la industria y la pequeña empresa son iguales de efectivas; sin embargo, la ICCM es más eficaz en el control de costos y gastos de administración y venta, pero menos eficiente en el uso de los activos en comparación con la PCCM. La ICCM y PCCM son más efectivas que la мссм, la primera por ser mucho más eficaz en el control de costos y gastos de admón y venta, y la segunda no solo por ser un poco más eficaz en la gestión de estos costos y gastos, sino ser más eficiente en el uso de los activos (tabla 15).

Tabla 15. Comparación de los indicadores de efectividad de la industria

\begin{tabular}{lllllllll}
\hline Indicador & $\mathbf{2 0 1 0}$ & $\mathbf{2 0 1 1}$ & $\mathbf{2 0 1 2}$ & $\mathbf{2 0 1 3}$ & $\mathbf{2 0 1 4}$ & $\mathbf{2 0 1 5}$ & $\mathbf{2 0 1 6}$ & Promedio \\
\hline & & & & & ICCM & & & \\
$\operatorname{ROA}(\%)$ & 3.3 & 5.4 & 5.0 & 3.6 & 4.8 & 4.0 & 7.7 & 4.8 \\
$\operatorname{ROE}(\%)$ & 6.3 & 8.6 & 8.0 & 5.4 & 5.4 & 5.0 & 10.6 & 7.0 \\
& & & & & $\mathrm{PCCM}$ & & & \\
$\operatorname{ROA}(\%)$ & 5.3 & 4.1 & 4.7 & 1.8 & 2.1 & 6.1 & 9.3 & 4.8 \\
$\operatorname{ROE}(\%)$ & 6.5 & 3.2 & 8.2 & 5.5 & 0.1 & 6.6 & 12.2 & 6.0 \\
& & & & & MCCM & & & \\
$\operatorname{ROA}(\%)$ & -0.5 & 3.3 & 4.5 & 5.3 & 4.8 & 4.6 & 2.2 & 3.5 \\
$\operatorname{ROE}(\%)$ & 2,9 & 5,0 & 2,9 & 5,7 & 3.7 & 4.0 & 1.9 & 3.7 \\
\hline
\end{tabular}

Fuente: elaboración propia con base en las tablas 2 y 9. 
Además, cuando se confronta el roe promedio de la ІССм (7.0\%), la РсСм (6.0\%) y la мССм (3.7\%), la ventaja es para la Iссм que logra ser más eficaz en el control de todos los costos y gastos que la РСсм y la мСсм, no obstante la РсСм logra reducir esa competencia con una mayor eficiencia en el manejo de activos y un mayor apalancamiento financiero positivo. Pero, por el contrario, esta superioridad aumenta en relación con la мссм por tener esta última un menor apalancamiento financiero. La superioridad del ROE de la РССм sobre MсCM se explica por su mayor eficiencia en la gestión de activos y utilizar un mayor apalancamiento financiero positivo (tablas 15 ).

\subsection{Valor económico agregado}

El Eva total de la ICCM es negativo en todos los años evaluados, lo mismo sucede con la рссм у la мссм. La destrucción del еva de la Іссм es creciente a partir del 2011, en la IPCD el EVA negativo baja en los primeros cuatro años, luego sube, y en la MCCM crece la destrucción de valor hasta 2013, posteriormente decrece (tabla 16, partes a, b y c).

Tabla 16. Comparación del eva promedio por empresa de la industria y sus inductores

\begin{tabular}{|c|c|c|c|c|c|c|c|c|}
\hline & 2010 & 2011 & 2012 & 2013 & 2014 & 2015 & 2016 & Promedio \\
\hline & \multicolumn{8}{|c|}{ a. EVA: ICCM } \\
\hline EVA (MM\$) & -292 & -100 & -217 & -366 & -510 & -733 & -821 & -434 \\
\hline UODI/ ANO (\%) & 5.9 & 9.9 & 9.0 & 5.5 & 7.4 & 6.4 & 10.7 & 7.8 \\
\hline Ko (\%) & 12.7 & 11.9 & 12.8 & 9.7 & 12.1 & 14.5 & 15.0 & 12.7 \\
\hline \multirow[t]{2}{*}{ ANO (MM\$) } & 4290 & 5113 & 5659 & 8857 & 10716 & 9040 & 18965 & 8949 \\
\hline & \multicolumn{8}{|c|}{ b. EVA: PCCM } \\
\hline EVA (MMUS\$) & -52 & -53 & -59 & -88 & -126 & -100 & -69 & -78 \\
\hline UODI/ANO (\%) & 9.3 & 6.8 & 7.8 & 2.6 & 3.0 & 8.9 & 10.4 & 7.0 \\
\hline Ko (\%) & 16.7 & 14.2 & 15.8 & 11.1 & 13.8 & 17.2 & 16.0 & 15.0 \\
\hline \multirow[t]{2}{*}{ ANO (MM\$) } & 700 & 727 & 750 & 1035 & 1165 & 1205 & 1239 & 974 \\
\hline & \multicolumn{8}{|c|}{ C. EVA: MCCM } \\
\hline EVA (MM\$) & -746 & -473 & -438 & -190 & -409 & -623 & -710 & -513 \\
\hline UODI/ANO (\%) & $-0,6$ & 4.4 & 6.0 & 7.2 & 6.3 & 6.3 & 3.3 & 4.7 \\
\hline Ko (\%) & 14,8 & 13.9 & 14.7 & 11.0 & 13.1 & 16.7 & 15.0 & 14.2 \\
\hline \multirow[t]{2}{*}{ ANO (MM\$) } & 4828 & 4961 & 4986 & 5055 & 5984 & 5962 & 6027 & 5401 \\
\hline & \multicolumn{8}{|c|}{ d. EVA del capital propio: ICCM } \\
\hline EVA de capital propio (MM\$) & -372 & -319 & -441 & -503 & -880 & -1108 & -866 & -641 \\
\hline ROE (\%) & 6.3 & 8.6 & 8.0 & 5.4 & 5.4 & 5.0 & 10.6 & 7.0 \\
\hline $\operatorname{Ke}(\%)$ & 15.9 & 15.2 & 16.6 & 12.7 & 16.0 & 20.6 & 19.3 & 16.6 \\
\hline Patrimonio (MM\$) & 3879 & 4843 & 5129 & 6871 & 8293 & 7096 & 9991 & 6586 \\
\hline
\end{tabular}




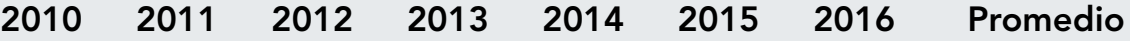

\begin{tabular}{lcccccccc} 
& $\mathbf{2 0 1 0}$ & $\mathbf{2 0 1 1}$ & $\mathbf{2 0 1 2}$ & $\mathbf{2 0 1 3}$ & $\mathbf{2 0 1 4}$ & $\mathbf{2 0 1 5}$ & $\mathbf{2 0 1 6}$ & Promedio \\
\hline & & \multicolumn{7}{c}{ e. EVA de capital propio: PCCM } \\
\hline EVA de capital propio (MM\$) & -59 & -70 & -57 & -53 & -133 & -123 & -48 & -77 \\
\hline ROE (\%) & 6.5 & 3.2 & 8.2 & 5.5 & 0.1 & 6.6 & 12.2 & 6.0 \\
\hline Ke (\%) & 20.4 & 17.1 & 18.5 & 12.4 & 16.2 & 20.0 & 16.8 & 17.3 \\
\hline Patrimonio (MM\$) & 427 & 507 & 553 & 770 & 829 & 923 & 1.050 & 723 \\
\hline & & & & f. EVA de capital propio: MCCM & & \\
\hline EVA de capital propio (MM\$) & -439 & -345 & -469 & -241 & -499 & -676 & -168 & -405 \\
\hline ROE (\%) & 2.9 & 5.0 & 2.9 & 5.7 & 3.7 & 4.0 & 1.9 & 3.7 \\
\hline Ke (\%) & 16.8 & 15.5 & 16.9 & 12.6 & 15.4 & 20.1 & 17.9 & 16.5 \\
\hline Patrimonio (MM\$) & 3174 & 3278 & 3343 & 3494 & 4273 & 4215 & 3868 & 3664 \\
\hline
\end{tabular}

Nota: MM\$ denota cifras en millones de pesos colombianos.

Fuente: elaboración propia e información de las tablas 3, 10, 11 y 13.

Al revisar los inductores del Eva total se encuentra que la UODI/ANO de la ICCM oscila en casi todos los años; mientras que en la РсCм varía hasta 2013, luego aumenta, y en la мссм crece hasta 2013 y luego decrece.

El Ko en la ICCM oscila hasta 2013, y luego aumenta, algo parecido ocurre en la PCCM y la мСсм, pero con una caída en 2016. El ANO de la ІССм, de la РССм y de la мСсм crece anualmente durante el septenio, pero con una caída en la Iссм en 2015 (tabla 16, partes a, b y c).

De estos tres grupos, la ICCM presenta la mayor rentabilidad de activo neto operacional $(7.8 \%)$ y el menor costo de capital (12.7\%), mientras que la мссм muestra la menor rentabilidad del ANO (4.7\%), y un costo de capital intermedio (14.2\%); lo que da como resultado que la pérdida residual promedio sea más alta en la мссм (-9.5\%), seguida de la РсСм $(-8.0 \%)$ y de la mostrada por la ІсСм $(-4.9 \%)$.

El Eva del capital propio fue negativo en todos los años en la Іссм, la Рссм y la мссм, con una tendencia a crecer entre 2012-2015 en la ICCм y una variabilidad a lo largo del periodo en la РСCM у MCCM, siendo más frecuente en esta última (tabla 16, partes d, e y f). Al revisar sus factores determinantes, se nota que el ROE sigue tendencias similares al EVA del capital propio en estos tres grupos; el Ke sigue una orientación contraria al EvA de capital propio en la ICCM y la MCCD en todo el periodo, y en algunos años en la PCCM. El patrimonio aumenta en todo el periodo en la РССм y la ICCM, pero con una caída en 2015 de esta última, mientras que la мссм el crecimiento va hasta 2014, luego cae. 
De los tres grupos, la ІССм ofrece una mayor rentabilidad a sus propietarios ( seguida de la РССм con $6.0 \%$ y la мСсм con $3.7 \%$; pero estos exigen un retorno superior en la РССм $(\mathrm{Ke}=17.3 \%)$ comparado con lo requerido en la ІсСм $(16.6 \%)$ y en la мССм $(16.5 \%)$. Aunque en ninguno de los tres grupos se logra superar las expectativas de los dueños, el que más se aleja de esta posibilidad es la мссм con una diferencia entre el roE y Ke promedios de $-12.8 \%$, y el más cercano es la IсCм con $-9.6 \%$.

\section{Conclusiones}

Del análisis realizado a las pymes del sector cuero, calzado y marroquinería en el periodo 2010-2016, se encontró que los activos y ventas promedio de las pequeñas y medianas empresas tienden a crecer, mientras que las utilidades netas oscilan en el periodo. Este comportamiento es similar al presentado por toda la industria de cuero, calzado y marroquinería.

Las pymes de este sector mostraron resultados contables favorables durante el periodo analizado al presentar, en promedio, utilidades netas y rendimientos positivos. Sin embargo, la рссм es más efectiva que la мссм en el logro de utilidades sobre la inversión, ya sea medida mediante el ROA o el RoE; esto se debe a la ventaja que saca la PCCM sobre la мссм en los siguientes factores, citados en orden de relevancia: 1) Eficiencia en el uso de activos, 2) Mantener siempre un apalancamiento financiero positivo y 3) Eficacia en la gestión de costos y gastos de producción y venta.

No obstante, la efectividad de la РССм y la мСсм еs superada por la ІсСм si se evalúa con el ROE, y solo es igualada por la РCCM si se mide con el ROA. Esto se debe a que la ICCM presenta una mayor eficacia en la gestión de todos los costos y gastos en comparación a la РсСм y a la мссм, además de contar con mayor grado de apalancamiento financiero que la мссм.

Los resultados positivos del desempeño financiero de las pymes de este sector, estimados por los indicadores contables, no son corroborados por los indicadores de valor económico agregado, dado que se encuentra que tanto la pequeña, como la mediana 
empresa del sector cuero, calzado y marroquinería en Colombia destruyen eva en todos los años, acumulando un saldo negativo del vma. Esta destrucción de valor obedece a que se genera un margen de pérdida residual en todos los años en las pymes de este sector.

Caso similar sucede con la Іссм que arroja resultados contables favorables, como utilidad neta, ROA y ROE, pero que destruye Eva en los siete años evaluados y genera un vma negativo. Esto es consecuencia de presentar un índice UODI/ANO inferior al Ko en todos los años.

En términos absolutos y relativos la мссм destruye mayor Eva que la Рссм.

Al revisar el eva de capital propio, se observa que la ІсСм, la РССм у la мссм destruyen valor a los propietarios, porque el rendimiento promedio del capital es menor al costo de capital propio. El grupo que más se aproxima a cumplir las expectativas de los dueños es la ICCDM, donde tiene participación las grandes empresas, mientras que la мсCм es la que más se aleja de este propósito.

En este trabajo de investigación se presentaron los factores que han influido en el desempeño de las pymes del sector cuero, calzado y marroquinería en Colombia, sin embargo, este estudio podría complementarse con investigaciones similares para grupos de empresas de la misma edad y ubicación geográfica en el ámbito nacional y con países donde se tienen acuerdos de libre comercio.

\section{Referencias}

Colombia, Congreso de la República. Ley 905 de 2004. Por medio de la cual se modifica la Ley 590 de 2000 sobre promoción del desarrollo de la micro, pequeña y mediana empresa colombiana y se dictan otras disposiciones (02 ago 2004).

Damodaran, A. (2017). [Base de datos en línea]. Recuperado de http://pages.stern.nyu.edu/ adamodar/

Dane (2012). Clasificación industrial internacional uniforme de todas las actividades económicas. Revisión 4 adaptada para Colombia. CIIU Rev. 4 A.C. Recuperado de https://www. dane.gov.co/files/nomenclaturas/CIIU_Rev4ac.pdf 
Dane (2017a). Encuesta Anual Manufacturera 2016. Anexos principales variables. Recuperado de https://www.dane.gov.co/index.php/estadisticas-por-tema/industria/encuesta-anual-manufacturera-enam

Dane (2017b). Encuesta Anual Manufacturera (EAM). Periodos de referencia: 2015, 2014, 2013, 2012, 2011 y 2010. Anexos. Recuperado de https://www.dane.gov.co/index.php/ estadisticas-por-tema/industria/encuesta-anual-manufacturera-enam/eam-historicos

Martín, J. \& Petty, J. (2001). La gestión Basada en el Valor. La respuesta de la empresa a la revolución del accionista. Barcelona: Ediciones Gestión 2.000 S. A.

Milla, A. (2010). Creación de valor para el accionista. España: Ediciones Diaz de Santos.

Modigliani, F. \& Miller, M. (1963). Corporate income taxes and the cost of capital: a correction. The American Economic Review, (53), 433-443.

Programa de Transformación Productiva (2018). ¿Qué es el sector cuero, calzado y marroquinería? Recuperado de https://www.ptp.com.co/ptp-sectores/historico/cuero-calzado-marroquineria

Rivera, J. (2004). Introducción a la administración financiera: fundamentos y aplicaciones para crear valor. Cali: Universidad del Valle.

Rivera, J. \& Alarcón, D. (2012). El cargo de capital en la evaluación del desempeño financiero de empresas innovadoras de confecciones de Cali. Estudios Gerenciales, 38(123), 85-100.

Rivera, J. \& Duque, C. (s. d.). Evaluación financiera de la industria del cuero, calzado y marroquinería en Colombia 2010-2016. [inédito].

Ross, S., Westerfield, R., \& Jordan, B. (2014). Fundamentos de finanzas corporativas (10a. edición). México: McGraw-Hill Interamericana.

Stewart, B. (2000). En busca del valor. Barcelona: Ediciones Gestión 2000.

Superintendencia de Sociedades (2017). Base de datos SIREM. Estados financieros y gastos de intereses. Recuperado de http://www.supersociedades.gov.co/asuntos-economicos-y-contables/estudios-y-supervision-por-riesgos/SIREM/Paginas/default.aspx

Superintendencia Financiera de Colombia (2017). Tasa de interés y desembolsos por modalidad de crédito. Recuperado de https://www.superfinanciera.gov.co/jsp/loader.jsf?IServicio=Publicaciones\&lTipo=publicaciones\&lFuncion=loadContenidoPublicacion\&id=60955

Wild, J., Subramanyam, K., \& Hasley, R. (2007). Análisis de estados financieros (9. ${ }^{a}$. edición). México: McGraw-Hill Interamericana.

Anexo: Pymes del sector cuero, calzado y marroquinería en Colombia 


\begin{tabular}{|c|c|c|c|}
\hline NIT & Razón social & 2010 & 2011 \\
\hline 800000604 & Orrego Valencia s.A.S. & 890212736 & Chic marroquineraia Ltda. \\
\hline 800025208 & Giorgio Sport S.A. & 890923902 & Comfer S.A. \\
\hline 800030780 & Mezclas de cauchos Ltda. & 900310790 & Tesluc S.A.S. \\
\hline 800031660 & Calzado Aliatti Ltda. & 900334029 & Curtidos Leathercol S.A.S. \\
\hline 800044194 & Ind. Mande partes Ltda. & 900370262 & Jem Supplies S.A.S. \\
\hline 800058369 & Inversiones y valores Wilcorts Ltda. Cl & 900436389 & Safety 2011 s.A.S. \\
\hline 800078522 & Fábrica de calzado Rómulo Ltda. & 900452281 & Tubos industriales y suelas S.A.S. \\
\hline 800098258 & Industrias Aquiles S.A.s. & 900472031 & Colombian leather imp \& exp s.A.S. \\
\hline 800098557 & Industria Roggers Lda. & 900531626 & C.I. gasaco imp. exp. S.A.S. \\
\hline 800100698 & Prefabricados D'marca Ltda. & 830120857 & C.I. inpieles S.A.S. \\
\hline 800123954 & Imapieles Ltda. & 900768031 & Upper saga de Colombia S.A.S. \\
\hline 800125786 & Bags Kiing Ltda. & 830016231 & Mas dotaciones y CIA Ltda. \\
\hline 800129600 & Manufacturas Beltrani Ltda. & 830097948 & Grupo GFC S.A.S. \\
\hline 800157508 & Manufacturas Af s.A.S. & 900214489 & Inversiones J \& D y A S.A.S. \\
\hline 800170877 & D L T Ltda. & 900224856 & Interpelli S.A.S. \\
\hline 800173338 & Emp. colomb. prod cuero de Caimán S.A. Cl. & 860071112 & Indalpe Ltda. \\
\hline 800191484 & Plantiformas S.A. & 800220701 & Formiplass S.A. \\
\hline 800196632 & Artesa S.A. & 811018390 & Pomelos S.A.S. \\
\hline 800219222 & Manufacturas Rical Ltda. & 830034002 & Oleaginosas del Ocoa S.A. \\
\hline 800226422 & Calzado Fratello Ltda. & 830096510 & Valores Smith S.A. \\
\hline 800242987 & D'lapel S.A. & 830097948 & Megainsumos S.A.S. \\
\hline 800252092 & Raiders Ltda. & 830081836 & Incopiel S.A.S. \\
\hline 802000027 & Curtiembres Camaguey S.A.S. & 830137213 & Phyton soles Ltda. \\
\hline 804010597 & Calzado Parisotto & 830500435 & Amada Colombia s.A.S. \\
\hline 804014026 & Suministros, transporte y tecnología S.A. & 860025954 & Frigorífico la gloria S.A.S. \\
\hline 805012644 & Calzado Golani Ltda. & 890103697 & Frigorífico la Parisienne S.A. \\
\hline 805020305 & Arriba International Shoes y CIA Ltda. & 860353254 & Oleaginosas de Colombia Ltda. \\
\hline 805020838 & JCT empresarial S.A. & 860508043 & Proalpet S.A. Cl \\
\hline 805022430 & Odena colombiana S.A. & 860512173 & cı Ind. Proc. de sebos y pieles nal. Itda \\
\hline 806004404 & c. I. curtiembres Matteucci S.A.S & 860529042 & Palmeras del Humea S.A. \\
\hline 807008358 & Calzado Burgos Ltda. & 890333047 & Conalplas S.A. \\
\hline 811004793 & Calza 3 s.A.S. & 900012114 & Cueros y más s.A.S. \\
\hline 811015541 & Colombiana de cueros S.A. Cl & 900173896 & Idecal Ltda. \\
\hline 811019447 & Schonthal international S.A. SUCUR CBIA & 900246695 & Agrosolera S.A. \\
\hline 811025865 & Product. de insumos para calzado s.A. & 900256495 & Frigotun S.A.S. \\
\hline 811037820 & Solsuole s. A. & 900493962 & Rawhide Products S.A.S. \\
\hline 811040924 & Industrias San Clemente S.A. & 900518732 & Samsonite Colombia S.A.S. \\
\hline 811041296 & Colvex Ltda. & 830029689 & Chaba S.A.S. \\
\hline 811044231 & Calzado JB & 860038140 & Product alimenticios Simonetta Itda \\
\hline 811046869 & Nigoon S.A. & 890205962 & Carnes y salsamentaria Baviera Itda \\
\hline
\end{tabular}




\begin{tabular}{|c|c|c|c|}
\hline NIT & Razón social & 2010 & 2011 \\
\hline 816000922 & Confecciones Argos Ltda. & 800220701 & Formiplass S.A. \\
\hline 817000170 & Almar marroquinera SCI S.A. & 900203470 & Manufacturas softwalk Ltda. \\
\hline 830002645 & Shoes Class Ltda. & 800158985 & Export alliance Ltda. \\
\hline 830002650 & Fábrica colombiana de moldes Ltda. & 800200760 & Fellinzi Ltda. \\
\hline 830003668 & Manufacturas Varomi Ltda. & 830055229 & Cuero y moda natural Skin Ltda. \\
\hline 830004799 & Baena mora y CIA Ltda. & 830106138 & Cyclus S.A.S. \\
\hline 830014770 & W sense Ltda. & 890302925 & Jack t galewski \& CIA Ltda. \\
\hline 830027229 & Ferplast Ltda. & 890900107 & Concuero S.A. \\
\hline 830043085 & Geca tannery s.A.s. & 900169735 & Tratos \& negocios E.U. \\
\hline 830046859 & Calzatore Hemisi Ltda. & 900300969 & Si de hongdesheng Colombia Ltda. \\
\hline 830053564 & Manufacturas Gonzo S.A. & 800085026 & Curtipieles S.A.S. \\
\hline 830055950 & Temac leather Ltda. & 805012644 & Calzado Golani s.A.S. \\
\hline 830058692 & Suelas y derivados de poliuretano Ltda. & 807008358 & Calzado burgos S.A.S. \\
\hline 830068579 & Manufacturas en cuero v \& c Ltda. & 807008358 & Manufacturas Beltrani S.A.S. \\
\hline 830073269 & Internacional de suelas Ltda. & 804010597 & Calzado Parisotto Ltda. \\
\hline 830081836 & Incopiel Ltda. & 811044231 & Calzado S.A.S. \\
\hline 830085645 & Industrias de caucho ZAC Ltda. & 890939566 & Montre S.A.S. \\
\hline 830085692 & Inversiones ponti C.I. Ltda. & 900394141 & Portafolio enermin S.A.S. \\
\hline 830089109 & Pro c pieles Ltda. & 800252066 & Colombian golf center Ltda. \\
\hline 830091710 & Lisardiny S.A. & 811000543 & C.I. arcoline S.A.S. \\
\hline 830101416 & Main colombia S.A. & 811019355 & Inyecciones y accesorios Ltda. \\
\hline 830101585 & Calzado siete cueros S.A. & 830144347 & Maquilagro S.A. \\
\hline 830108567 & United shoe E.U. & 830145629 & Gravatti S.A.s. \\
\hline 830119873 & C.I. manufacturas y moda Ltda. & 900283560 & C.I. vetra trading S.A.S. \\
\hline 830133822 & Tecnimold Ltda. & 900333380 & Intertes sur S.A.S. \\
\hline 830137387 & Sotercueros S.A. & 900401718 & Tan Colombia s.A.S. \\
\hline 830137489 & Dart leather Ltda. & 800043202 & Del llano S.A. \\
\hline 830141456 & C.I. exporth leather S.A. & 800058242 & Salsamentaria inducolcarnes S.A.S. \\
\hline 830505384 & Curtiembres la gaviota Ltda. & 800092777 & Grupo nova S.A. \\
\hline 830505432 & Creaciones Raferty CIA Ltda. & 800097832 & Juguetes caninos S.A. \\
\hline 830505522 & Tecnimolplast Ltda. & 800186295 & Gustar S.A.S. \\
\hline 830515234 & Inver H y L Ltda. & 830034787 & Aceites y concentrad del Caribe Itda. \\
\hline 860000918 & Sucesores de Wolf Kerpel Ltda. & 800187325 & C.I. expopieles del caribe S.A.S. \\
\hline 860027512 & Fábrica de calzado Palermo Ltda. & 890904603 & Fábrica de calzado setenta S.A. \\
\hline 860033182 & Industria manufacturera de calzado Ltda. & 890912464 & Alpha shoes S.A. \\
\hline 860053373 & Calzado Atlas Ltda. & 890922271 & Artículos de cuero Ltda. \\
\hline 860054922 & Rali Ltda. & 890924742 & Fábrica de calzado Kondor Ltda. \\
\hline 860055508 & Curtiembres el reno s.A. & 890928871 & Inversiones Arango Tamayo S.A.S. \\
\hline 860057178 & Facalve Ltda. & 890934628 & Arte en piel Ltda. Arpiel \\
\hline 860066651 & Saquirsal \& CIA. Ltda. & 890936742 & Calzado noval Ltda. \\
\hline 860353338 & Manufacturas volare S.A. & 900006622 & Tihany S.A. \\
\hline
\end{tabular}




\begin{tabular}{llll}
\hline \multicolumn{1}{c}{ NIT } & \multicolumn{1}{c}{ Razón social } & $\mathbf{2 0 1 0}$ & \multicolumn{1}{c}{$\mathbf{2 0 1 1}$} \\
\hline 860500924 & Calzado infantil colniã'os Ltda. & 900026171 & Curtiembres alianza leather Ltda. \\
\hline 860504811 & Mai Ltda. & 900049881 & Zarzarrossa Ltda. \\
\hline 860509812 & Colombo italiana de curtidos Ltda. & 900061263 & Curtilether S.A. \\
\hline 860512396 & Manufacturas de cuero Beltman Ltda. & 900064154 & C.I. manufacturas quintero S.A. \\
\hline 860521924 & Cueros y artíc. Marroquin. tipo export. Itda & 900086028 & Calzados 3025 S.A. \\
\hline 860523238 & Fábrica de calzado Gerama Ltda. & 890104728 & C.I. modapiel S.A. \\
\hline 860532076 & Química I.T. Ltda & 890109177 & Maquiplast Ltda. \\
\hline 890000758 & Trianon S.A. & 890200236 & Curtiembres del valle Ltda. \\
\hline 890003011 & Curtimbres Sierra Pérez y CIA S. en C. & 890205628 & lii milenio S.A. \\
\hline 890101952 & Plasticron S.A. & 890305571 & La maravilla S.A. \\
\hline 890900244 & Mesace S.A. & 890504493 & Mussi zapatos S.A.S. \\
\hline
\end{tabular}

Fuente: elaboración propia con base en Sirem (Superintendencia de Sociedades, 2017) y Rivera \& Duque (2017). 